\title{
Reference values for a fetal movement acceleration measurement recorder to count fetal movements
}

\author{
Eiji Ryo ${ }^{1}$, Hideo Kamata ${ }^{1}$, Michiharu Seto ${ }^{1}$, Masayoshi Morita ${ }^{1}$, Yohei Nagaya ${ }^{1}$, Kyoko Nishihara ${ }^{2}$ and Noboru Ohki ${ }^{3}$
}

BACKGROUND: A newly developed fetal movement acceleration measurement recorder has made it possible to count gross movements for hours. The purpose of this study was to determine the normal reference values for such movements. METHODS: One hundred and six pregnant women recorded fetal movements by themselves when they slept at home weekly from 28 weeks to term. The normal reference values were determined based on the data that could be recorded for more than $4 \mathrm{~h}$ per night.

RESULTS: A total of 2,458 h of data from 385 recordings from 64 women was available. The median ratio of 10-s periods in which fetal movements occurred to the total time interval was $17 \%$ at 28 gestational weeks, decreasing to $\sim 6 \%$ at term. The number of fetal movements was 74 times/h, decreasing to 29 times at term. The number, the mean, and the longest durations of periods with no fetal movement, meaning no fetal movements were found for more than $5 \mathrm{~min}$, were 1.56 times/ h, 7.95 and $14.25 \mathrm{~min}$, respectively, at 28 weeks, and increasing to 2.54 times, and 9.63 and 19.67 min, respectively, at term.

CONCLUSIONS: This study provides normal reference values for gross fetal movement count using the fetal movement acceleration measurement recorder.

$\mathbf{F}$ etal movements have long been recognized as an important biological index to estimate the state of a fetus. Fetal movements that are observed on ultrasonography are important indices on biological and physical scoring systems to determine the well-being of a fetus (1). These scoring systems have substantially contributed to the field of perinatal medicine; however, ultrasonography only allows for a short observation time in medical facilities and not at home.

Counting fetal movements is easy for the mother to do anytime and anywhere, and it is recommended by some medical facilities; however, there has been no agreement as to whether maternal counting improves perinatal outcomes. There have been insufficient data to guide us regarding the management of decreases in fetal movements detected by maternal counting (2). Furthermore, maternal counting is subjective and also not suitable for long periods of time.
Some recent reports have indicated the potential of ultrasonography to observe fetal movements as an index of the neurological developments of fetuses. Kurjak et al. (3) proposed a new scoring system for fetal movements observed by three-/ four-dimensional ultrasonography to detect fetal brain impairment. Morokuma et al. (4) also devised an antenatal behavior test by ultrasonography to assess fetal brain development. However, these tests have not become commonly used.

Despite the potential of informative indices of fetal movements, they cannot be fully used because there has been no practical and objective method to count fetal movements for longer periods at home.

We have been developing a fetal movement acceleration measurement recorder (FMAM recorder, http://e-mother.cosite.jp) for counting gross fetal movements at home. In a preliminary study (5), we simultaneously observed gross fetal movements using ultrasonography and oscillations of the mother's abdomen using the FMAM recorder, and demonstrated that these two methods have a high level of correlation. The recorder is designed to enable recording gross fetal movements for many hours not only at medical facilities but also at home. In another preliminary study (6), the mothers were able to successfully record fetal movements for hours at home. Furthermore, an original computer software system for the FMAM recorder has been developed to automatically analyze and count fetal movements. We found a high agreement between manual and automated signal analysis, meaning the new software can be used to accurately count fetal movements and successfully minimize the number of artifacts (7). The FMAM recorder has made it possible for mothers to count gross fetal movements for many hours at home. However, the utility of the FMAM recorder has been limited because there are no normal reference values for fetal movements.

The purpose of this study was to use the FMAM recorder to obtain normal reference values for fetal movements.

\section{METHODS}

\section{Subjects}

A total of 106 singleton pregnant women who had no medical complications and were taking neither psychotropic nor hormonal

\footnotetext{
'Department of Obstetrics and Gynecology, School of Medicine, Teikyo University, Itabashi-ku, Tokyo, Japan; ${ }^{2}$ The Institute for Science of Labour, Shibuya-ku, Tokyo, Japan; ${ }^{3}$ NoruPro Light Systems, Kokubunji-shi, Tokyo, Japan. Correspondence: Eiji Ryo (yonchi@med.teikyo-u.ac.jp)

Received 11 August 2017; accepted 18 December 2017; advance online publication 17 January 2018. doi:10.1038/pr.2017.328
} 
medication participated in the study. All women underwent routine examinations during pregnancy at Teikyo University Hospital.

\section{FMAM recorder}

The FMAM recorder (Figure 1) has been explained in detail elsewhere (6). It contains two acceleration sensors and weighs $290 \mathrm{~g}$. One sensor is a fetal movement sensor (FM sensor) that is placed on the mother's abdominal wall, and the other is a mother's movement sensor (MM sensor) placed on her thigh. The FM and MM sensors are similar in structures but have different sensitivities (FM: $700 \mathrm{mV} /$ $0.1 \mathrm{G}, \mathrm{MM}$ : $120 \mathrm{mV} / 0.1 \mathrm{G}$ ). Each sensor is disk-shaped, $20 \mathrm{~g}$ in weight, and $2.8 \mathrm{~cm}$ in diameter. It has two electrodes with capacitive acceleration, of which one is a movable diaphragm, and the other is a fixed back plate. The diaphragm has a slight weight, which works as a pendulum; this increases its sensitivity in detecting oscillation. A change in acceleration appears as the amount of change in delta $C$ in electrostatic capacity $C$ between the diaphragm and the back plate arising from the displacement of the diaphragm. They are noninvasive.

The FM sensor detects oscillations of the mother's abdominal wall caused by fetal movements. However, the mother's movements also cause oscillations. The recorder is unsuitable for use when the mother moves frequently, which is why it is used when the mother is sleeping. However, the mother occasionally moves during her sleep, and the MM sensor detects these movements. In principle, when the MM sensor does not detect the mother's body movement and the FM sensor detects abdominal wall oscillations, gross fetal movements are judged to have occurred.

\section{Fetal movement recording}

The FMAM recorder can be operated by the woman herself at home. The subjects were instructed on how to use the recorder and then took it home. The instruction took around $10 \mathrm{~min}$. Before sleeping, the mother attached the sensors to her abdomen and thigh using
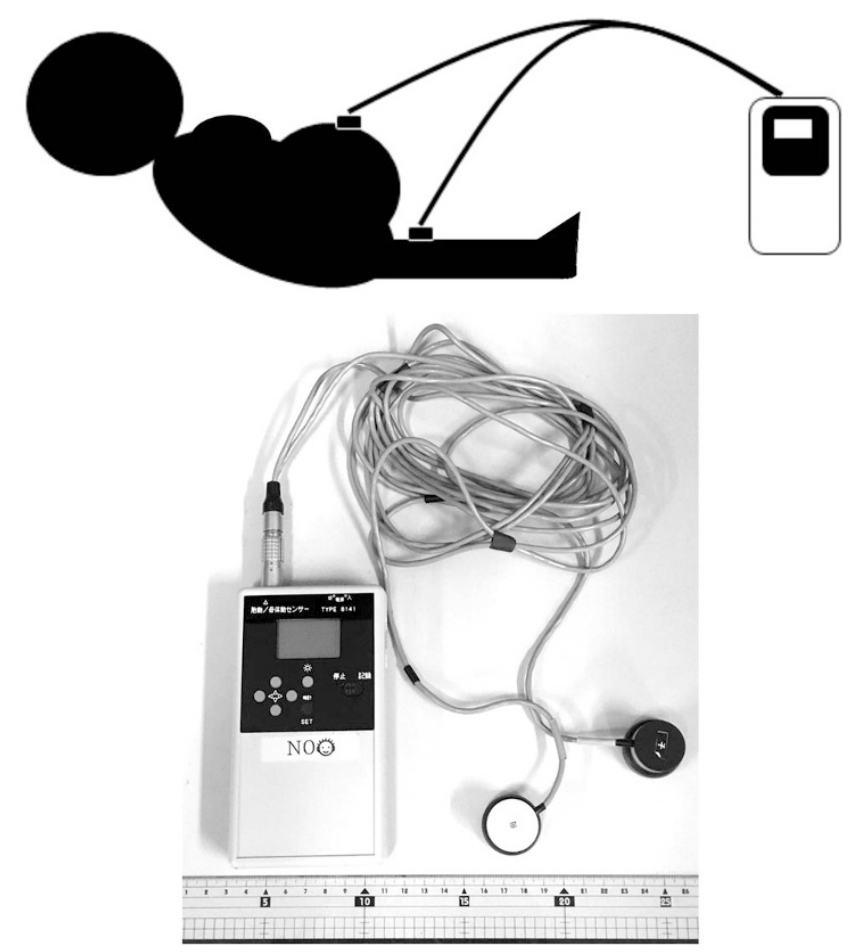

Figure 1. Fetal movement acceleration measurement recorder. The recorder consists of two capacitive acceleration sensors. One is a fetal movement sensor placed on the maternal abdominal wall, and the other is a mother's movement sensor placed on her thigh. adhesive and surgical tape. Next, she turned on the recorder and fell asleep. The following morning, she woke up and turned off the FMAM recorder. The data from that night were recorded on a Secure Digital card.

The mothers were asked to record fetal movements weekly from 28 weeks to term because our previous study (5) demonstrated that the agreements between the oscillations detected by the FMAM recorder and gross fetal movements observed by ultrasonography were almost perfect at 28-39 weeks. Every time the mother visited the hospital for a routine checkup, she brought the Secure Digital card and the two sensors. The data on the card were transferred to a computer. The sensors were checked by a calibrator that was developed for checking the sensors' sensitivity. Then, the card and sensors were given back to her for the next recording.

\section{Data analysis}

All data transferred to the computer were first analyzed using the newly developed software system (version 1.04A), and some revisions were made using its manual functions. These procedures have been explained in detail elsewhere (7). We will introduce them briefly here:

Preprocessing: Figure 2 shows a screen shot of the automatic analysis for a woman at 30 gestational weeks. (a) At the top shows the maternal (upper) and fetal (lower) acceleration signals from 1:30 AM to 8:10 AM. The Arabic numerals indicate time. A red vertical line is shown around 4:50 AM, and a magnified view of the same time period is shown in (b). The top line shows the maternal raw acceleration signal after being filtered with a Butterworth filter. The low-pass digital filter was cut off at $2.5 \mathrm{~Hz}$ $(-12 \mathrm{~dB} /$ oct $)$ and a high-pass filter was cut off at $0.5 \mathrm{~Hz}(-6 \mathrm{~dB} /$ oct). The signal was then changed to absolute integral values per $50 \mathrm{~ms}$, which are shown in the second line. Similarly, the third and fourth lines show the fetus's raw acceleration signals and their integral values, respectively.

Detecting signals: Both maternal and fetal signals were analyzed the same way. When the integral values were greater than twice the average amplitude during $3 \mathrm{~s}$ just before and after measurement, they were judged to be positive for acceleration. This procedure was useful for excluding various noises. Positive maternal accelerations are shown by the red rectangles at the top of the magnified view in (b). Maternal and fetal accelerations are shown by red and blue rectangles on the judgment line, which is (c). When an interval between two positive accelerations is more than $1 \mathrm{~s}$, the accelerations are judged to be two movements, and when the interval is $<1 \mathrm{~s}$, they are together counted as one movement.

Excluding periods of maternal activity or wakefulness: Any period in which the MM sensor detected positive accelerations more than four times per minute was deleted from the data because this usually indicated that the mother was active or awake, e.g., going to the restroom. Any such period is shown as a light blue rectangle in (a). Moreover, when there were several active periods and the interval between them was $<5 \mathrm{~min}$, the interval was also judged to be an active or awake period and deleted manually. Excluding maternal body movement during sleep: Even when the mother is sleeping, all positive accelerations detected by the FM sensor do not indicate fetal movements. Accelerations caused by the mother need to be deleted. When the FM sensor and the MM sensor detected accelerations at the same time, it was judged to be maternal movements, such as changing body position. These are expressed as red rectangles in (c). When the MM sensor does not detect the mother's body movement and the FM sensor detects abdominal wall oscillations, fetal movements are judged to have occurred, and these are expressed as a blue rectangle in (c).

Excluding maternal respiration: Automatic exclusion of the mother's respiration has not been completed yet, and manual exclusion is still needed. However, a minimum threshold amplitude level can be set at various levels and is helpful to reduce manual work. In a previous study (7), the default values were maternal level 20 and fetal level 15. In this study, we also set 


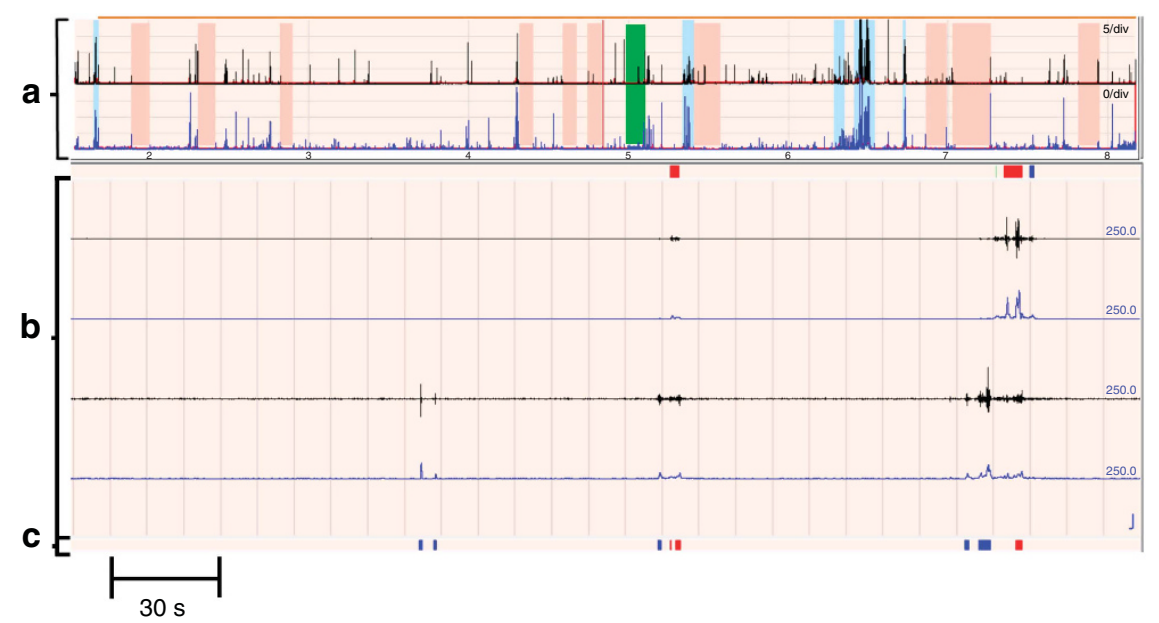

Figure 2. A screen shot of the automatic analysis software showing the details for a pregnant woman at 30 gestational weeks. (a) A general view of the maternal and fetal acceleration signals in the upper and lower parts, respectively, between 1:30 and 8:10 AM. A detail of the time indicated by the vertical red line is shown in a magnified view in (b). The first line shows the raw maternal acceleration signals, and the second shows their integral values. The third and fourth lines show the raw fetal signals and their integral values, respectively. The decisions on how to count the maternal and fetal movements are shown as red and blue rectangles, respectively, in (c).

the fetal level at 20. Maternal respiration, including snoring, has various patterns but is regular in principle and can be identified. Excluding fetal hiccups: Fetal hiccups are sudden, quick fetal body movements at regular intervals and are recognized as physiological phenomena. They are different phenomena from gross movements and are not counted as gross movements in the fetal biophysical profile used worldwide (1). In our previous study (5), we simultaneously observed fetal hiccups by ultrasonography and the following maternal abdominal wall oscillations with the acceleration sensor. Based on that study, characteristic regular accelerations at 15-20 beats/min were defined as the sign of fetal hiccups. Fetal hiccups usually persist for several minutes as a bout of fetal hiccups, and these data are not counted as fetal movements. A bout of fetal hiccups is shown as a yellow-green rectangle in (a). The usual duration and frequency of fetal hiccups were reported in another study (8).

In summary, fetal movements were defined as irregular positive accelerations, except for accelerations caused by the mother's body movements. The blue rectangles in (c) indicate fetal movements.

\section{Normal reference values}

Fetal movement counting started when the mother fell asleep and ended when she woke up. The time of falling asleep was defined as the start of a 30-min period in which the MM sensor did not detect more than four accelerations per minute. It was determined by a manual review combined with the software analyses. The time of waking up was when the recorder was turned off. We used the data for this research only when a record could be obtained for more than $4 \mathrm{~h}$ per night, because we thought that is near the minimum sleeping time for normal pregnant women. Reference values were determined based on data for women who had a normal pregnancy and delivery.

Of the original 106 women, 85 uneventfully delivered normalweight, full-term newborns without anomalies or neurological problems. The births took place at Teikyo University Hospital between February 2010 and July 2016; 21 of those had recordings that were not properly recorded for more than $4 \mathrm{~h}$. Therefore, the recordings from 64 women were available. The normal references (median and 10-90\%) were determined with the use of JMP Pro 12.0.1, depending on the number of gestational weeks.

The recording was divided into 10-s time intervals; thus, 360 intervals/h. Next, all intervals (total time) were reviewed. An interval was judged to be positive for fetal movements (a positive interval) when a blue rectangle was present. Thereafter, the ratio of the number of positive intervals to the total time for one night was calculated.

The number of blue rectangles, which indicated fetal movements, was counted, and their average number per hour was calculated for one night.

A period of no-fetal movement period was defined as when no blue rectangles were found for more than $5 \mathrm{~min}$. These periods are shown as pink-colored rectangles in Figure 2 (a). The average number per hour and the average and longest duration of these periods throughout one night were calculated. Using the positive interval number, the following first and second supplementary values were examined.

The recording for one night was divided equally into the early, middle, and late night periods. Then, the three percentages of positive intervals in each period to the all-night value were calculated. Using the three percentages, we determined one triangular diagram point. Next, the gestational periods were classified into three periods such as $28-31,32-35$, and $36-$ 39 weeks because it has been unclear whether the distribution of fetal movement occurrence changes constantly as pregnancy progresses. After that, the points were plotted into each triangle diagram.

To examine whether normal values for male and female subgroups were needed, the positive interval ratios for each were compared. Furthermore, fetal movements are known to continually decrease as pregnancy progresses; thus, each pregnancy was divided into the early (28-33 weeks) and late periods (3439 weeks), and the ratios were compared again for both periods. Comparisons were made using the Wilcoxon's method, and differences with a $P$ value $<0.05$ were considered statistically significant.

This study was approved by the ethical committee of Teikyo University. All mothers gave their written informed consent for participation.

\section{RESULTS}

Table 1 shows the characteristics of the 64 pregnant women and newborns. A total of 385 records were available, and the total recording time was $2,458 \mathrm{~h}$. The mean (standard deviation) recording time for one night was 6.39 (1.2) h. No 
negative side effects were noted, except for mild contact dermatitis caused by the adhesive tape.

The results were as follows:

Figure $3 \mathbf{a}$ shows the reference values for the positive interval ratios. The median was $\sim 17 \%$ at 28 gestational weeks, decreasing to $\sim 6 \%$ at term. The decrease was not linear from 28 to 39 weeks, but it seemed more rapid around $33-35$ weeks.

Figure $\mathbf{3 b}$ shows the values for the average number of fetal movements per hour. The median number of fetal movements was 74 at 28 gestational weeks, decreasing to 29 at term. The decreasing pattern was similar to that of the positive interval ratios.

Figure 4 shows periods with no fetal movement by week of gestation: (a) the mean number per hour, (b) the mean duration per night, and (c) the longest duration per night. The median of (a), the mean number per hour was 1.56 at 28 weeks, increasing to 2.54 at term. The mean duration (b) and the longest duration (c) at 28 weeks were 7.95 and $14.25 \mathrm{~min}$, respectively. They increased to 9.63 and $19.67 \mathrm{~min}$, respectively, at term. The increase in (a) was more rapid during weeks $32-36$.

Table 1. Characteristics of pregnant women and newborns

\begin{tabular}{lc}
\hline Variable & Number or mean (range) \\
\hline Mother & 64 \\
Age (years) & $32.7(20-44)$ \\
BMI & $21.0(16.8-28.0)$ \\
Primipara/multipara & $43 / 21$ \\
Delivery weeks & $39.1(37.0-41.1)$ \\
Vaginal/cesarean delivery & $46 / 18$ \\
Newborn & 64 \\
Weight (g) & $2,974.1(2,292.0-3,790.0)$ \\
Male/female & $31 / 33$ \\
pH of umbilical artery blood & $7.279(7.083-7.506)$ \\
\hline
\end{tabular}

BMI, body mass index

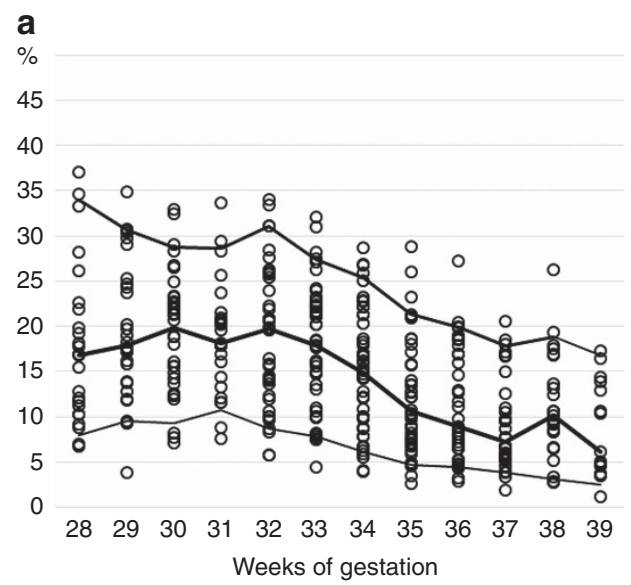

Figure 5 shows triangular diagrams for 28-31, 32-35, and 36-39 gestational weeks. The diagram for 28-31 weeks shows most dots clustered near the center. This indicates that fetal movements occurred more equally during the three periods each night. However, the dot locations expanded as the pregnancy progressed, indicating that fetal movements became more unequally distributed. The mean recording time was $\sim 6 \mathrm{~h}$, which indicated that counting for $2 \mathrm{~h}$ was sufficient for the earlier stages of pregnancy in most cases, but was insufficient and could show inaccurate counting numbers for the later stages.

The medians of the ratios for male and female fetuses at 28-39 weeks were $15.4 \%$ and $14.3 \%$, respectively, indicating no significant difference $(P=0.91)$. Similarly, these ratios were $18.5 \%$ and $17.9 \%$ at $28-33$ weeks, and $10.1 \%$ and $10.9 \%$ at $34-39$ weeks, respectively. There were no significant differences in either periods $(P=0.87,0.94$, respectively). Based on these results, there was no need for different normal values for males and females.

\section{DISCUSSION}

We performed this study with the FMAM recorder to obtain normal reference values for fetal movement. Overall, the ratios of the number of positive to total epochs decreased during 28-39 gestational weeks. The number of gross movements decreased in a similar pattern. The incidence, average duration, and longest duration of periods with no fetal movement increased after 28 weeks. In a supplementary study, we found that fetal movements occurred equally during early sleep, middle sleep, and late night sleep during the earlier stages of pregnancy; however, they sometimes diverged during later stages. There were no differences in gross fetal movements between male and female fetuses.

The biggest challenge of the study was determining the accuracy of the FMAM recorder during home use. For fully confirming the accuracy, it would be necessary to simultaneously observe fetal movements using the FMAM recorder

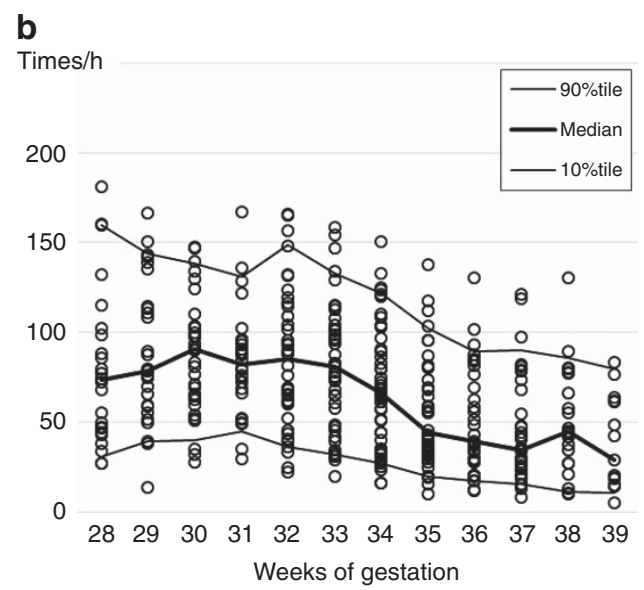

Figure 3. Changes in the gross fetal movement count by the number of gestational weeks. (a) The ratio of positive interval numbers to total time. (b) The average number of fetal movements per hour throughout one night. 
a

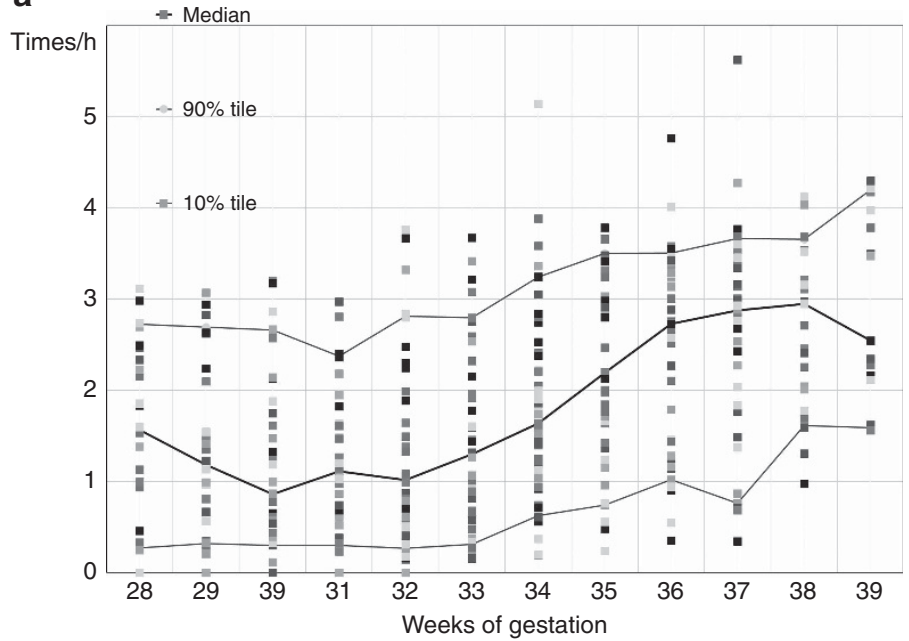

b

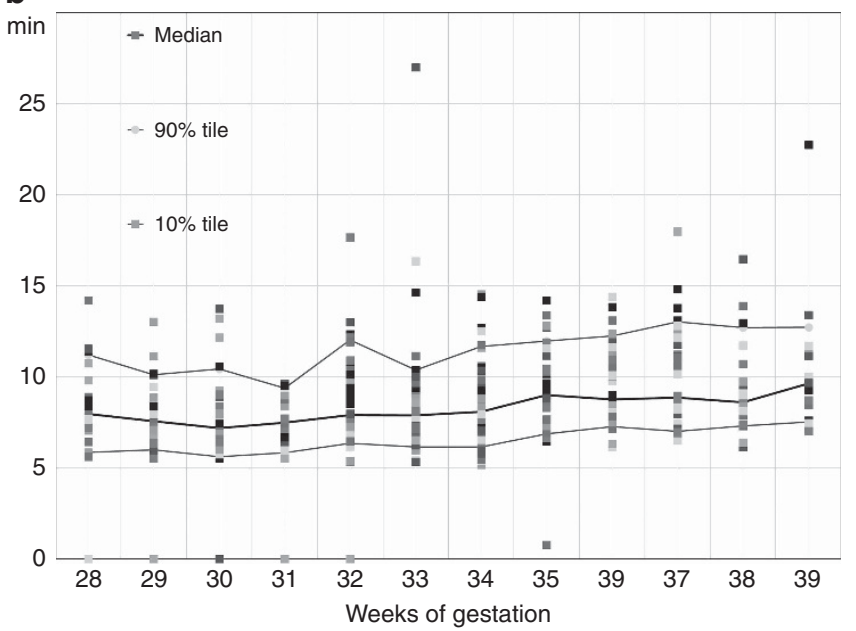

C

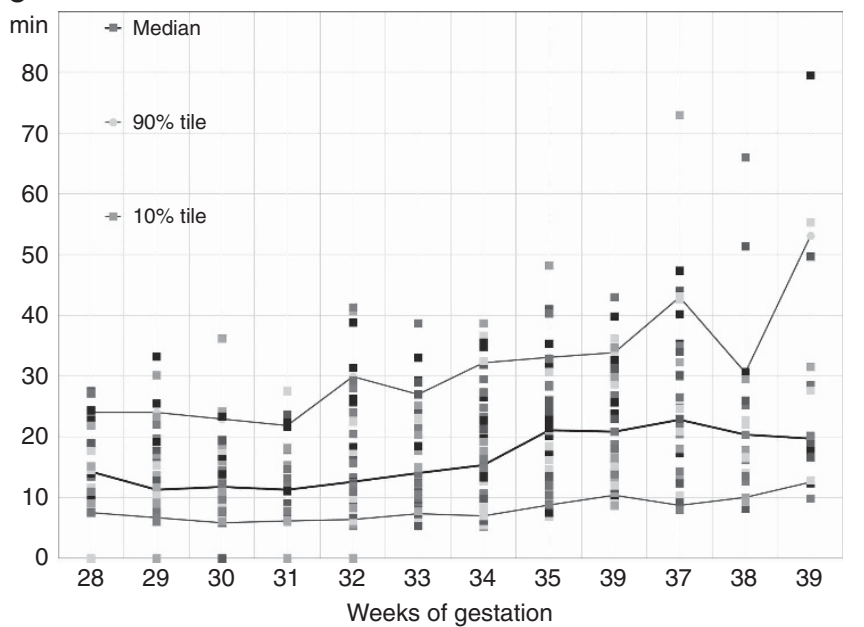

Figure 4. Changes in periods of no fetal movement by the number of gestational weeks. (a) The average number of times per hour, (b) the average duration, and (c) the longest duration throughout one night.
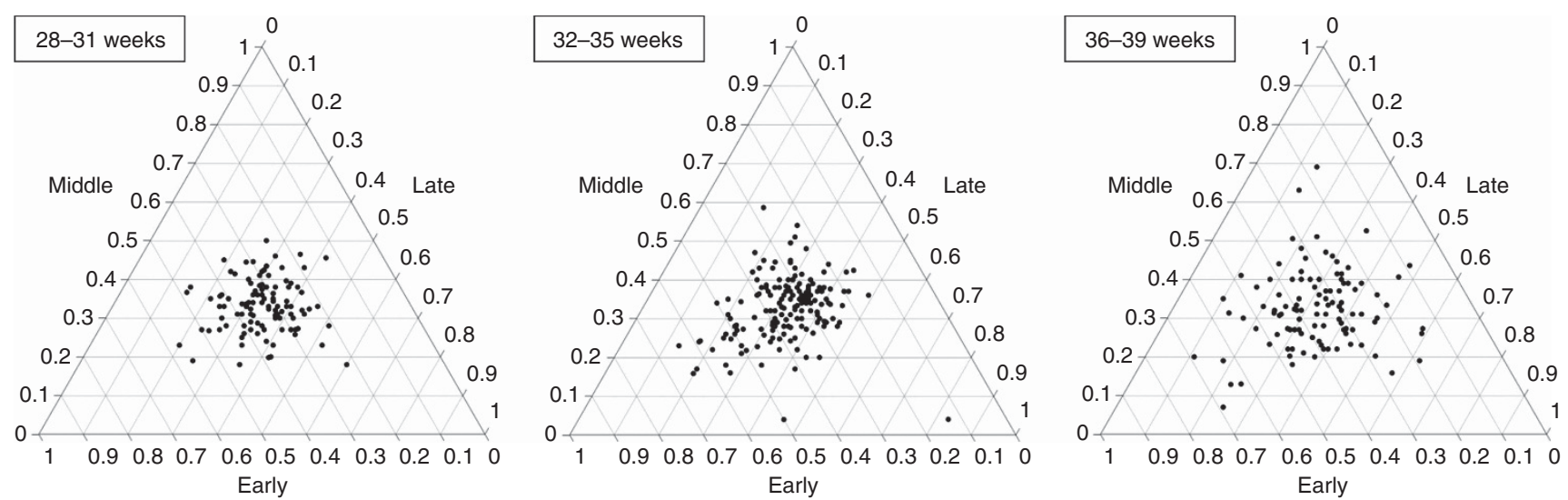

Figure 5. Triangular diagrams of the ratio of positive intervals to the total at $28-31,32-35$, and 36-39 gestational weeks. Each dot was determined based on the three percentages of the positive intervals divided by the all-night value for the early, middle, and late night periods. Dots in the center indicate that fetal movements were equally distributed throughout each night; dots away from the center indicate that these movements were unequally distributed. 


\section{Articles | Ryo et al.}

and ultrasonography while the mothers slept naturally and moved occasionally; however, this would be ethically and methodologically difficult to do. Methodologically, manual ultrasonography itself inevitably and continually causes abdominal wall oscillations that affect the FMAM recorder's measurements, whereas ultrasonography with a fixed probe cannot respond to the mother's movements.

In a previous study (5), we simultaneously observed maternal abdominal oscillations using the FMAM recorder and gross fetal movements using ultrasonography (the probe was fixed in place to avoid causing oscillation). The mothers kept quiet in a semi-Fowler position. The agreement between the two methods was almost perfect at 28-39 weeks. The recorder proved to be accurate when the mothers kept quiet. During home use of the recorder, however, the mothers occasionally move their body even when sleeping. In a previous experiment (5), we found that maternal arm, hand, or head movements without trunk movements did not influence the FM sensor, but leg movements influenced both the FM and MM sensors. In this study, the signals for fetal movements were counted only when the mothers did not move their leg. Therefore, the recorder may be reliable in theory; however, the accuracy of the FMAM recorder has not been still proven in practice.

To refer to the accuracy of the FMAM recorder from a practical viewpoint, the results obtained in this study were compared with those of several ultrasonographic studies (9-13).

Patrick et al. (9) reported that the mean percentage time during which gross movement existed at 34-35 weeks was $8.2 \%$. Separately, they also reported $9.8 \%$ at $34-35$ weeks and $11.2 \%$ at $38-39$ weeks (10). Another study reported $12.2 \%$ when delivery was within 3 days and $11.6 \%$ when delivery was more than 7 days away (11). Ten Hof et al. (12) provided a normal reference range for fetal body movements from observations of 29 normal fetuses. They observed fetal movements with ultrasonography for 60 min biweekly from 24 to 36 weeks and for 120 min weekly after 36 weeks of gestation. Their study indicated that the median percentage incidence of gross fetal movements decreased from $\sim 17 \%$ at 24 weeks to $7 \%$ near term, which was very similar to the changes in the positive interval ratios in our study, which indicated about $17 \%$ at 28 weeks and $6 \%$ at 28 near term.

Theoretically, the positive 10 -s interval ratio is not the same as the percentage of time in which gross movements occur. As in the signals shown in Figure 2, the duration of accelerations is much shorter than the duration of the gross movements themselves. Gross fetal movements produce acceleration of the abdominal wall only for a moment. One fetal rolling body movement is presumed to produce one short series of accelerations within one 10 -second period in most cases, although more complicated movements might produce accelerations that appear in two or more 10 -s periods. The duration of such movements was reported to be $\sim 7 \mathrm{~s}$ near term in a study by D'Ellia et al. (13). Even when movement lasting $<10 \mathrm{~s}$ caused oscillation, one 10-s interval was counted as positive in our study. Therefore, the ratio should be a little larger than the time percentage measures of movements. However, the FMAM recorder has less sensitivity for gross movements than ultrasonography, which has perfect sensitivity. The FMAM recorder should show a smaller number than ultrasonography because the agreement between the two instruments was $\sim 0.8$, as shown in our previous study (5). This might be why the results of our study were very similar to those of the study by Ten Hof et al. (12). The results of our study were presumed to be similar to the ultrasonographic studies (9-13).

Overall, we now assume that the FMAM recorder is capable of counting gross fetal movements at home; however, clinical prospective cohort studies will be needed to confirm whether the FMAM recorder is accurate and useful. Furthermore, several factors such as amniotic fluid volume, placental site, and maternal obesity may affect the counting, which needs further study.

One of the advantages of the FMAM recorder is that it can count fetal movements over a long time period. In this study, only recordings exceeding $4 \mathrm{~h}$ per session could be used. Fetuses do not move at a uniform pace; therefore, counting fetal movements may be inaccurate if the time period is insufficient. Ultrasonography has long been used to observe fetal movements; however, it is not practical for long time periods for most fetuses. Observing fetal movements by ultrasonography for $2 \mathrm{~h}$ is a strenuous procedure, and the first supplementary study showed that even this procedure might sometimes be insufficient in later stages of pregnancy. Furthermore, ultrasonography has bioeffects. The American Institute of Ultrasound in Medicine recommends that ultrasound exposure should be "as low as reasonably achievable" (14). In experiments with embryonic mice (15), exposure to ultrasound with the intensity of that used for diagnostic use for $30 \mathrm{~min}$ or longer affected neuronal cell migration in the cerebral cortex. It is still unclear whether long-term ultrasonography for many normal fetuses is acceptable. In comparison, the FMAM recorder is only a sensor and sends out no signal, which means that it has no bioeffect on either the mother or the fetus.

The second supplementary study was done to determine whether fetal movement differs between male and female fetuses. It is known that there are neurological differences between boys and girls after birth; however, little is known about the differences in neurological development of fetuses. Di Pietro et al. (16) found that male fetuses were more active than female fetuses at 38-39 gestational weeks. A study by Mediana et al. (17) found that male fetuses seemed to have a higher incidence of movement at term; however, the difference disappeared after adjustment for the effect of the fetal wakefulness cycle. They emphasized the importance of this adjustment and concluded that there were no sex-related differences. The FMAM recorder enabled a long observation period, and we directly obtained the same results without the need for adjustment. 
The number, mean duration, and longest duration of periods of no fetal movement increased as pregnancy progressed. A period of no fetal movement means not only fetal stillness but also the continuance of stillness, and this could be related to fetal rest-activity cycles. The increase might be a sign that central neurological developments are gaining control of the peripheral neuron system and decreasing the body movements. Such periods were defined when no blue rectangles were found for $5 \mathrm{~min}$, which might seem to be a relatively short duration. However, the percentage of positive intervals was $17 \%$ at 28 gestational weeks. If fetal movements occurred randomly, negative 10-s intervals (absence of movement) for five consecutive minutes (30 intervals) would be rare $(0.83(1-0.17)$ to the 30 th power equals 0.0037 , or $0.37 \%)$. We thought, therefore, that an absence of fetal movements for five consecutive minutes would be a nonrandom event and might have physiological significance. This study was laying the groundwork for the FMAM recorder, and from the beginning, we tried not to overlook any nonrandom event. Further study is needed to clarify whether a long absence of movement has any physiological significance.

As pregnancy progresses, fetal movements decrease and periods of no fetal movement increase. Interestingly, both the number of fetal movements and the periods of no fetal movement did not change linearly but changed rapidly at around 32-36 weeks. Nijhuis et al. (18) reported that the states of fetal behavior are not fully established before 36 weeks. The rapid changes in fetal movements at around 32-36 weeks might be related to the development of fetal behavioral states.

The FMAM recorder has a great deal of potential. Decreased fetal movements precede a stillbirth in many cases (19). Counting fetal movements with the recorder may contribute to fewer stillbirth. Decreasing fetal movement is reportedly related to many fetal complications such as fetal growth restriction, non-reassuring fetal status, umbilical cord complication, placental insufficiencies, preterm birth, oligohydramnios, congenital malformation, chromosomal abnormalities, fetal brain injury, fetomaternal transfusion, intrauterine infection, and hypoglycemia (20), which all need further study, and the FMAM recorder could assist with these studies. Furthermore, little is known about fetal rest-activity movement cycles. Several researchers demonstrated that the emergence of fetal behavioral states is delayed in fetal growth restriction $(21,22)$. From birth to peripuberty, the rate of cyclic alternating pattern, which is an endogenous rhythm during non-rapid eye movement sleep, has been reported to decrease in children with disabilities such as Down syndrome, autism, Asperger syndrome, and attention-deficit/hyperactivity disorder (23). Is there any possibility that these disabilities are related to abnormal fetal rest-activity movement cycles? The recorder could expand horizons in these research areas.

There are certain limitations with the FMAM recorder, though. The software analysis system cannot yet completely exclude noise caused by maternal respiration. The recorder is unsuitable for clinical use in the daytime when the mother moves frequently. We could ask the mother to sit still during the recording time in the daytime which might be one way to use the FMAM recorder, but that would cause inconvenience when the recording time is long. Furthermore, some women have complained that the adhesive tape caused contact dermatitis, and others said that the cables between the recorder and sensors were troublesome. We think it would be better to make the sensor wearable and wireless. Regarding the principal limitations, the recorder can assess only quantitative but not qualitative movements of the fetus. As for infants, a quality assessment of general movement has become an important predictor of neurological developments (24). The combination of ultrasonography and the FMAM recorder might be needed for qualitative and quantitative assessments.

The current FMAM recorder and its software system for analysis are still prototypes and need many improvements; however, we think that this situation is similar to the early stages of ultrasonography. We believe that the FMAM recorder will contribute greatly to the field of perinatal medicine in the near future.

\section{ACKNOWLEDGMENTS}

We thank Professor Takuya Ayabe and Mieko Fuse. We also appreciate the cooperation of the women who participated in this study.

STATEMENT OF FINANCIAL SUPPORT: THIS WORK WAS SUPPORTED BY JSPS KAKENHI (GRANT NUMBER JP 16K10109).

Disclosure: The authors declare no conflict of interest.

\section{REFERENCES}

1. Manning FA, Platt LD, Sipos L. Antepartum fetal evaluation: development of a fetal biophysical profile. Am J Obstet Gynecol 1980;136:787-95.

2. Hofmeyr GJ, Novikova N. Management of reported decreased fetal movements for improving pregnancy outcomes. Cochrane Database Syst Rev 2012;18:CD009148.

3. Kurjak A, Miskovic B, Stanojevic M, et al. New scoring system for fetal neurobehavior assessed by threeand four-dimensional sonography. J Perinat Med 2008;36:73-81.

4. Morokuma S, Fukushima K, Yumoto Y, et al. Simplified ultrasound screening for fetal brain function based on behavioral pattern. Early Hum Dev 2007;83:177-81.

5. Ryo E, Nishihara K, Matsumoto S, Kamata H. A new method for longterm home monitoring of fetal movement by pregnant women themselves. Med Eng Phys 2012;34:566-72.

6. Ryo E, Kamata H. Fetal movement counting at home with a fetal movement acceleration measurement recorder: a preliminary report. J Matern Fetal Neonatal Med 2012;25:2629-32.

7. Nishihara K, Ohki N, Kamata H, Ryo E, Horiuchi S. Automated software analysis of fetal movement recorded during a pregnant woman's sleep at home. PLoS ONE 2015;10:e0130503.

8. Kamata H, Ryo E, Seto M, Morita M, Nagaya Y. Counting fetal hiccups using a fetal movement acceleration measurement recorder. J Matern Fetal Neonetal Med 2017;30:475-8.

9. Patrick J, Fetherston W, Vick H, Voegelin R. Human fetal breathing movements and gross fetal body movements at weeks 34 to 35 of gestation. Am J Obstet Gynecol 1987;130:693-9.

10. Patrick J, Campbel K, Carmichael L, Natale R, Richardson B. Patterns of fetal body movements over 24-hour observation intervals during the last 10 weeks of pregnancy. Am J Obstet Gynecol 1982;142:363-71. 


\section{Articles | Ryo et al.}

11. Carmichael L, Campbell K, Patrick J. Fetal breathing, gross fetal body movements, and maternal and fetal heart rates before spontaneous labor at term. Am J Obstet Gynecol 1984;148:675-9.

12. Ten Hof J, Nijhuis IJ, Mulder EJ, et al. Longitudinal study of fetal body movements: nomograms, intrafetal consistency, and relationship with episodes of heart rate patterns a and B. Pediatr Res 2002;52:568-75.

13. D'Elia A, Pighetti M, Moccia G, Santangelo N. Spontaneous motor activity in normal fetuses. Early Hum Dev 2001;65:139-47.

14. Fowlkes JBBioeffect Committee of the American Institute of Ultrasound in Medicine. American Institute of Ultrasound in Medicine consensus report on potential bioeffects of diagnostic ultrasound. J Ultrasound Med 2008;27:503.

15. Ang ES Jr, Gluncic V, Duque A, Schafer ME, Rakic P. Prenatal exposure to ultrasound waves impacts neuronal migration in mice. Proc Natl Acad Sci USA 2006;103:12903-10.

16. DiPietro JA, Costigan KA, Shupe AK, et al. Fetal neurobehavioral development: association with socioeconomic class and fetal sex. Dev Psychobiol 1998;33:79-91.
17. Medina PG, Visser GH, Huizink AC, et al. Fetal behavior does not differ between boys and girls. Early Hum Dev 2003;73:17-26.

18. Nijhuis JG, Prechtl HF, Martin CB Jr, Bots RS. Are there behavioural states in the human fetus? Early Human Dev 1982;6:177-95.

19. Whitty JE, Garfinkel DA, Divon MY. Maternal perception of decreased fetal movement as an indication for antepartum testing in a low-risk popuration. Am J Obstet Gynecol 1991;165:1084-8.

20. Frpen JF, Tveit JVH, Saastad E, et al. Management of decreased fetal movements. Semin Perinatol 2008;32:307-11.

21. Arduini D, Rizzo G, Caforio L, Boccolini MR, Romanini C, Mancuso S. Behavioural state transitions in healthy growth retarded fetuses. Early Hum Dev 1989;19:155-65.

22. van Viet MA, Martin CB Jr, Nijhuis JGPrechtl HFR. Behavioural states in growth-retarded human fetuses. Early Hum Dev 1985;12:183-97.

23. Bruni O, Novelli L, Miano S, Parrino L, Terzano MG, Ferri R. Cyclic altenating pattern: a wondow into pediatric sleep. Sleep Med 2010;11:628-36.

24. Prechtl HFR, Einspieler C, Cioni G, Bos AF, Ferrari F, Sontheimer D. An early marker for developing neurological deficits after perinatal brain lesions. Lancet 1997;349:1361-3. 